\title{
Steganography in Audio Files by Hermite Transform
}

\author{
Sandra L. Gomez-Coronel ${ }^{1,2, *}$, Boris Escalante-Ramírez ${ }^{1}$, Marco A. Acevedo-Mosqueda ${ }^{3}$ and Ma. Elena Acevedo \\ Mosqueda ${ }^{3}$ \\ ${ }^{1}$ Departamento de Procesamiento Digital de Señales, Universidad Nacional Autónoma de México, México D.F. \\ 2 Academia de Electrónica, UPIITA IPN, México D.F. \\ ${ }^{3}$ Sección de Estudios de Posgrado e Investigación, ESIME Zacatenco Telecomunicaciones, IPN, México D.F.
}

Received: 6 May. 2013, Revised: 10 Sep. 2013, Accepted: 11 Sep. 2013

Published online: 1 May. 2014

\begin{abstract}
This work proposes the use of the Hermite Transform (HT) for imprinting audio files with hidden information. One of the most important aspects of this kind of concealment derives from the amount of data that a host file (audio, images, data, or video) can hold. In this particular case, both the host and the hidden files are audio files. The amount of information that can be hiddenwith the suggested technique-occupies half the length of the host file. Furthermore, the use of the Hermite Transform on audio files helps improve the algorithm performance. Experimental results show that our proposal is efficient and effective, because the audio file concealment is imperceptible to human hearing. Performance of our proposal was assessed using correlation and peak signal to noise ratio. The results strengthen the effectiveness of the method.
\end{abstract}

Keywords: Audio files, Hermite transform, Signal processing, Steganography

\section{Introduction}

Although Internet is a medium that enables both communication and data transmission through different means-audio, video, texts, and images-, and is employed in many applications, it cannot guarantee the integrity of information during the transmission process; hence it can be corrupted. Since most applications require transmit data in a confidential manner, many security measures have become quite important, functioning as a basis for new techniques related to the protection of data. One of them is steganography [1], which is a technique used for concealing a message that needs to be kept a secret within a public channel: the message goes unnoticed to those individuals that are not aware of the specific data, thus allowing the imperceptible transmission of large quantities of information. Images, audio, or video can be used for masking information in order to ensure important information.

The data secretly hidden or sent is called concealed information, while the digital file hosting is known as host or carrier. In the present paper, both the concealed information and the host are audio files.

Many methods for the concealment [1,2] of audio files have been developed. However-and without taking into account the technique employed-it is important to consider the following elements: perceptive invisibility (the concealed information must go unnoticed to the audience; in this case, hearing); robustness (resistance present in the utilized technique when facing the carrier?s manipulation, such as compression or filtering); and capacity (amount of information that can be hidden in the host without affecting the rest of its characteristics).

A simple technique for hiding data is through the modification of the Least Significant Bits [3]. It requires the modification of those bits whose contribution to the carrier signal are less valuable. Although it constitutes a simple technique, and the changes in the host file are hardly perceptible to the human ear, it is sensible to alterations, which translates into low robustness. It is important to mention that various techniques have been developed, based on the LSB alteration, so as to improve the quantity of bits that can be modified [9], which allows for larger quantities of data to be concealed.

Another technique is proposed in [4], called spread spectrum. In this technique the audio is coded throughout the whole frequency range. This allows the audio to be transmitted over different frequencies that vary according to the spectrum extension method employed. Those techniques that utilize this type of methodology are the

\footnotetext{
*Corresponding author e-mail: sgomezc@ipn.mx
} 
safest for transmitting a hidden message in an audio file; nevertheless, random noise can lead to data loss.

The different developed techniques can be applied in the spatial and in the transform domain-Fourier Transform, Discrete Cosine Transform (DCT), or Discrete Wavelet Transform (DWT). In the present work, we proposed used the Hermite Transform.

The Hermite transform was originally proposed by Martens [5] and successfully used in [6,7]. This transform allows local processing on visual information. It also shows features that make it an efficient tool in graphic applications; it allows local processing on the visual information. It also incorporates the "masking" property of human visual system (HCS), it is based on Gaussian functions derivates [8], and is a particular case of the Polynomial transform.

As the Discrete Wavelet Transform (DWT), HT decomposes a signal into a number of coefficients, where the zero-order coefficients represent the average Gaussian measurement, and higher order coefficients contain details. In addition, these coefficients can be generated with and without subsampling. The coefficients containing the details are used to hide the audio file.

In this particular case, we used an audio file (*.wav) of 8 bits, (A carrier), with a sampling frequency of 44100 samples per second and Pulse Code Modulation (PCM). The audio to hide is also an audio file, with the same parameters, $W$.One of the advantages of this work is the amount of information hidden: if the original file size is $A(n, 2)$ ( $n$ : rows and 2 columns), then the amount of information that will be hidden is $W(n / 2,2)$.

The results showed that our proposal is an efficient method when the hidden information and the carrier are audio files. The advantage is that the hidden file results imperceptibly audible when it is embedded in the host audio. Besides, when it is recovered, there is not noticeable audible change, since the sample is very clear.

This paper is organized as follows: in section 2, the mathematical theory of the Hermite Transform is presented. Section 3 describes our proposal of hidden audio files. The experiments and results are presented in Section 4. Finally, the conclusions are detailed in Section 5 .

\section{The Hermite Transform}

The Hermite Transform $[5,6,7,8,10]$ constitutes a particular case of polynomial transform, which is a signal decomposition technique where the signals are locally approximated by polynomials.

\subsection{Polynomial Transform}

The analysis employing a polynomial transform involves two stages. In the first one, the original signal $L(x))$ is localized by multiplying it by a window function $V(x)$. A complete description of the signal requires the repetition of the localization process at a sufficient number of window positions. From the localized window function $V(x)$, it is possible construct a weighting function $W(x)($ Eq. 1) by periodic repetition:

$$
W(x)=\sum_{k} V(x-k T),
$$

where $T$ is the period.

Provided $W(x)$ is nonzero for all $x$, we get (Eq. 2):

$$
L(x)=\frac{1}{W(x)} \sum_{k} V(x-k T)
$$

Therefore, we are guaranteed that the localized signals $L(x) V(x-k T)$ contain enough information about the original signal, for all different window positions $k T$.

The second stage consists in approximating the signal segment-within the window-with a polynomial. As basis functions for the polynomial expansion, we use the polynomials $G_{n}(x)$ which are orthonormal regarding $V_{2}(x)$, where $\mathrm{n}$ is the degree of the polynomial as Eq. (3) shows:

$$
\int_{-\infty}^{\infty} V(x) G_{m}(x) G_{n}(x) d x=\delta_{n m}
$$

The orthonormal polynomials for an arbitrary window function $V_{2}(x)$ are given by Eq. (4):

$$
G_{n}(x)=\frac{1}{\sqrt{M_{n-1} M_{n}}}\left[\begin{array}{cccc}
c_{0} & c_{1} & \ldots & c_{n} \\
c_{1} & c_{2} & \ldots & c_{n+1} \\
\vdots & \vdots & \ldots & \vdots \\
c_{n-1} & c_{n} & \ldots & c_{2 n-1} \\
1 & x & \ldots & x^{n}
\end{array}\right]
$$

where the determinant $M_{n}$ is defined by Eq. (5):

$$
\begin{aligned}
M_{n} & =\left|c_{i+j}\right| \\
M_{-1} & =-1 \\
c_{n} & =\int_{-\infty}^{\infty} x^{n} V^{2}(x) d x
\end{aligned}
$$

If $V^{2}(x)$ is even, $c_{2 n+1}=0$ the expressions $G_{i}$ are simplified.

Under general conditions [11] for the original signal $L(x)$, by Eq. (6) we get that:

$$
V(x-k T)\left[L(x)-\sum_{n=0}^{\infty} L_{n}(k T) G_{n}(x-k T)\right]=0
$$

where $L_{n}(k T)$ is the direct polynomial transform and is defined by Eq. (7) as follows,

$$
L_{n}(k T)=\int_{-\infty}^{\infty} L(x) G_{n}(x-k T) V^{2}(x-k T) d x
$$


The coefficients $L_{n}(k T)$ can be derived from the signal $L(x)$ by convolving with the filter functions (8):

$$
D_{n}(x)=G_{n}(-x) V^{2}(-x)
$$

The inverse polynomial transform equations (2) and (6) is defined by Eq. (9):

$$
L(x)=\sum_{n=0}^{\infty} \sum_{k} L_{n}(k T) P_{n}(x-k T)
$$

where the pattern functions are defined by Eq. (10):

$$
P_{n}(x)=\frac{G_{n}(x) V(x)}{W(x)}
$$

The inverse polynomial transform consists of interpolating the coefficients $L_{n}(k T)$ with the pattern function $P_{n}(x)$ and summing over all orders $n$.

\subsection{Hermite Transform}

The important parameters that have to be considered in a polynomial transform are the window function and the sampling period. In HT, the applied window has a Gaussian distribution (11):

$$
V(x)=\frac{1}{\sqrt{\sqrt{\pi \sigma}}} \exp \left(\frac{x}{2 \sigma}^{2}\right)
$$

where $\sigma$ is the standard deviation of the Gaussian function.

The filter functions that are convolved signals are calculated by Eq. (12):

$$
D_{n}(x)=\frac{(-1)^{n}}{\sqrt{2^{n} n !}} \frac{(1)}{\sigma \sqrt{\pi}} H_{n}\left(\begin{array}{l}
x \\
\sigma
\end{array}\right) \exp \left(\frac{-x^{2}}{\sigma^{2}}\right)
$$

In the special case of the Gaussian window, we obtain the Hermite polynomials [12], which are defined by Eq. (13):

$$
H_{n}(x)=(-1)^{n} \exp \left(x^{2}\right) \frac{d}{d x^{n}} \exp \left(-x^{2}\right)
$$

The filter function $D_{n}(x)$, is equal to the nth order derivative of a Gaussian function defined by Eq. (14) as follows:

$$
D_{n}(x)=\frac{(-1)^{n}}{\sqrt{2^{n} n !}} \frac{d}{d\left(\frac{x}{\sigma}\right)^{n}}\left[\frac{1}{\sigma \sqrt{\pi}} \exp \left(\frac{-x^{2}}{\sigma^{2}}\right)\right]
$$

The Fourier transform is (15):

$$
d_{n}(\omega)=\frac{1}{\sqrt{2^{n} n !}}(j \omega \sigma)^{n} \exp \left(\frac{-\omega^{2} \sigma^{2}}{4}\right)
$$

The maximum value for $(\omega \sigma)^{2}$ is $2 n$; hence, filters of increasing order successively analyze higher frequencies in the signal. However, for large orders, the frequency peaks move very close together, therefore, sequential filters give only very little additional information.

The pattern functions $P_{n}(x)$ necessary to recover to the original signal from the coefficients of the Hermite transform are defined by Eq. (16).

$$
P_{n}(x)=\frac{T}{\sqrt{2^{n} n !}} \frac{1}{\sqrt{2 \pi}} H_{n}\left(\begin{array}{c}
x \\
\sigma
\end{array}\right) \frac{\exp \left(\frac{-x^{2}}{2 \sigma^{2}}\right)}{W(x)}
$$

\section{Proposed Algorithm}

As we indicated the HT decomposes a signal in different components. The total energy of the original signal is distributed in each of these components. The energy measurement [13] is important when an application of this type (steganography or watermarking) replaces or modifies information of the original signal, because it is important to prevent loss of energy. Therefore, the shortfall of a considerable amount of energy in a signal means that essential information is also lost. In all signals, a large percentage of energy is found at low frequencies. Considering this premise, it was decided to manipulate the components of lower energy for hiding the audio file.

An audio file (*.wav) of 8 bits, with a sampling frequency of 44100 samples per second and Pulse Code Modulation (PCM), was decomposed with HT to determine which were the components of lower energy. Subsequently, the energy of each component was calculated, in order to identify those with lower energy. This exercise was repeated with samples of $n$ audio files to determine if the distribution of energy was similar. Once this was verified, various tests were performed to establish the number of components that had to be modified so that the file hidden would stay imperceptible, allowing at the same time its retrieval.

The following sections describe the procedure for concealing and extracting the secret information.

\subsection{Hiding information}

1.The audio carrier is read. $A(n, 2)$ (n: rows and 2 columns).

2.The Hermite Transform coefficients $A(n, 2)$ are calculated in order to get: $\hat{A}\left(\frac{n}{2}, 2, n c\right)$.

3.The four coefficients of lower energy of $\hat{A}$ are determined, since this ensures redundancy, and especially, it guarantees that the secret information will be audibly imperceptible.

4.For each of the four components of lower energy, its maximum and minimum value is established for setting a threshold that is used in the concealment process of the audio file. The determination of each 
value of threshold is made per component and it corresponds to Eq. (17):

$$
\text { threshold }=\left|\max \left(\hat{A}\left(\frac{n}{2}, 2, n c\right)\right)-\min \left(\hat{A}\left(\frac{n}{2}, 2, n c\right)\right)\right|
$$

5.The audio signal to be hidden $S\left(\frac{n}{2}, 2\right)$ is read, and separated into two channels (left and right). Only the voice channel $S_{1}\left(\frac{n}{2}, 1\right)$ is chosen. It becomes clear that the amount of information that can be hidden in the host audio is $\frac{n}{2}$.

6.To hide the information in each of the Hermite coefficients of lower energy, we use the Eq. (18):

$$
\hat{A}\left(\frac{n}{2}, 1, n c\right)=\text { threshold } * S_{1}\left(\frac{n}{2}, 1\right)
$$

Each modified component will have its respective threshold.

7.The inverse Hermite Transform is calculated to have: $A_{\text {mod }}(n \times 2)$.

8. The new audio file is $A_{\text {mod }}$.

\subsection{Extracting information}

1.The audio signal, $A_{\text {mod }}$, is read.

2.The Hermite Transform coefficients are calculated to get: $\hat{A}_{\text {mod }}\left(\frac{n}{2}, \frac{m}{2}, n c\right)$.

3. We determined the four lower energy components from $\hat{A}_{\text {mod }}$.

4.Extract the hidden information of each low energy component, according to the Eq. (19):

$$
S_{\text {mod }}\left(\frac{n}{2}, 1\right)=\frac{\operatorname{Amod}\left(\frac{n}{2}, \frac{m}{2}, n c\right)}{\text { threshold }}
$$

Therefore, there are four recovery samples, and we can determine which contain the original file without audible changes.

\section{Experimental Results}

Our proposal was tested in fifty different audio files. First, an audio file was used as host and fifty different samples were concealed. Then fifty host audio files were modified with a single sample. We used correlation and Peak Signal to Noise Ratio to assess the quality or our proposal.

a) Correlation

The correlation $r$ indicates relation between two variables [13], in this case between the host audio and modified audio file (see equation 20).

$$
r=\frac{\sum_{m} \sum_{n}\left(A_{m n}-A\right)\left(B_{m n}-B\right)}{\sqrt{\left(\sum_{m} \sum_{n}\left(A_{m n}-A\right)^{2}\right)\left(\sum_{m} \sum_{n}\left(B_{m n}-B\right)^{2}\right)}}
$$

where:
$A$ : original signal
$B$ : carrier signal
$\hat{A}$ : original signal average
$\hat{B}$ : carrier signal average

b) Peak Signal to Noise Ratio

An objective way to get an answer to the file quality is the Signal to Noise Ratio (SNR) [14]. This relation is defined as the ratio between signal intensity and the noise intensity that accompanies it. For this particular case, the peak signal to noise ratio PSNR [15] was defined by Eq. (21):

$$
P S N R=10 \log _{10} \frac{N A^{2}}{\left\|A-A_{\text {mod }}\right\|^{2}}
$$

where $N$ is the length of the carrier signal, $A$ is the maximum absolute square value of the original signal, and $\left\|A-A_{\text {mod }}\right\|^{2}$ is the energy of the difference between the original and the carrier signal.

Table 1 shows the correlation and PSNR values of the original and the carrier signals, as well as, the correlation between the original and the recovered audio final.

The correlation of these components was the same; therefore only one value was included. Besides, all samples extracted from the three components were "audibly" well heard, without noticing that it had been modified. Regarding the audio signal, it is also clear that the correlation is high, as well as the PSNR value, indicating that there was no noise and did not change significantly. Regarding the audible aspect, neither change was noticed with any of the fifty embedded samples.

Figure 1 shows the original audio signal with its two channels. In figure 2 we show the audio signal modified and the original audio signal (only one channel). We only display a sample with length 882000 . According to the figures, it is clear that the based audio signal was not modified; consequently its information was not altered. The same applies to audio files that were used as samples.

Figure 3 shows the original audio file to hide and the recovered audio file. For this example we used the sample 7. It can be seen that the recovered sample is very similar to the original file. Once more, the results proved that inaudible modification is shown.

A very important factor to take into account is the amount of information hidden. Its length is exactly half the length of the host audio. It has a lot of information to be hidden without greatly altering the host audio. Besides, the samples can be recovered without significant changes. Another test was performed using fifty host audio files and a single audio file as a samples. In table 2, we can observe the values of correlation and PSNR. As before, for all the tests, the hidden audio and the recovered audio did not show any distortion. From Table 2, we can observe that both hidden information and recovered audio 
Table 1: Correlation and PSNR values from fifty different audio files to hide

\begin{tabular}{|c|c|c|c|}
\hline $\begin{array}{l}\text { Sample to } \\
\text { hide }\end{array}$ & $\begin{array}{l}\text { PSNR } \\
{[\mathrm{dB}]}\end{array}$ & $\begin{array}{l}\text { Correlation } \\
\text { Original and } \\
\text { modified file }\end{array}$ & $\begin{array}{c}\text { Correlation } \\
\text { Original and } \\
\text { extracted file }\end{array}$ \\
\hline 1 & 33.1887 & 0.9908 & 0.9638 \\
\hline 2 & 30.5079 & 0.9832 & 0.9797 \\
\hline 3 & 34.4267 & 0.9930 & 0.9503 \\
\hline 4 & 39.1526 & 0.9976 & 0.8659 \\
\hline 5 & 35.3001 & 0.9943 & 0.9431 \\
\hline 6 & 30.5214 & 0.9832 & 0.9805 \\
\hline 7 & 31.1130 & 0.9853 & 0.9770 \\
\hline 8 & 33.3408 & 0.9911 & 0.9632 \\
\hline 9 & 29.1681 & 0.9773 & 0.9847 \\
\hline 10 & 37.3177 & 0.9964 & 0.9118 \\
\hline 11 & 33.9464 & 0.9922 & 0.9580 \\
\hline 12 & 32.5547 & 0.9894 & 0.9689 \\
\hline 13 & 31.4957 & 0.9865 & 0.9742 \\
\hline 14 & 32.9015 & 0.9902 & 0.9664 \\
\hline 15 & 32.3370 & 0.9888 & 0.9692 \\
\hline 16 & 29.9594 & 0.9810 & 0.9818 \\
\hline 17 & 32.5839 & 0.9894 & 0.9690 \\
\hline 18 & 34.6857 & 0.9934 & 0.9498 \\
\hline 19 & 30.8211 & 0.9843 & 0.9791 \\
\hline 20 & 34.0892 & 0.9925 & 0.9562 \\
\hline 21 & 33.3773 & 0.9912 & 0.9628 \\
\hline 22 & 33.1490 & 0.9907 & 0.9611 \\
\hline 23 & 33.7856 & 0.9919 & 0.9594 \\
\hline 24 & 31.2930 & 0.9859 & 0.9766 \\
\hline 25 & 32.4109 & 0.9890 & 0.9699 \\
\hline 26 & 29.7428 & 0.9800 & 0.9825 \\
\hline 27 & 32.9798 & 0.9903 & 0.9651 \\
\hline 28 & 30.8443 & 0.9844 & 0.9781 \\
\hline 29 & 34.2274 & 0.9927 & 0.9553 \\
\hline 30 & 37.3821 & 0.9964 & 0.9106 \\
\hline 31 & 29.0532 & 0.9767 & 0.9856 \\
\hline 32 & 28.8593 & 0.9757 & 0.9859 \\
\hline 33 & 27.4246 & 0.9666 & 0.9901 \\
\hline 34 & 36.7365 & 0.9959 & 0.9220 \\
\hline 35 & 32.1196 & 0.9883 & 0.9708 \\
\hline 36 & 37.0117 & 0.9961 & 0.9165 \\
\hline 37 & 28.2829 & 0.9724 & 0.9874 \\
\hline 38 & 35.1583 & 0.9941 & 0.9446 \\
\hline 39 & 34.5502 & 0.9932 & 0.9517 \\
\hline 40 & 29.1105 & 0.9770 & 0.9842 \\
\hline 41 & 33.2302 & 0.9909 & 0.9638 \\
\hline 42 & 32.4925 & 0.9892 & 0.9694 \\
\hline 43 & 37.6069 & 0.9966 & 0.9051 \\
\hline 44 & 31.7822 & 0.9873 & 0.9737 \\
\hline 45 & 32.5207 & 0.9893 & 0.9691 \\
\hline 46 & 32.1006 & 0.9882 & 0.9718 \\
\hline 47 & 32.9583 & 0.9903 & 0.9654 \\
\hline 48 & 34.1504 & 0.9926 & 0.9556 \\
\hline 49 & 33.4728 & 0.9914 & 0.9622 \\
\hline 50 & 29.6188 & 0.9795 & 0.9840 \\
\hline
\end{tabular}

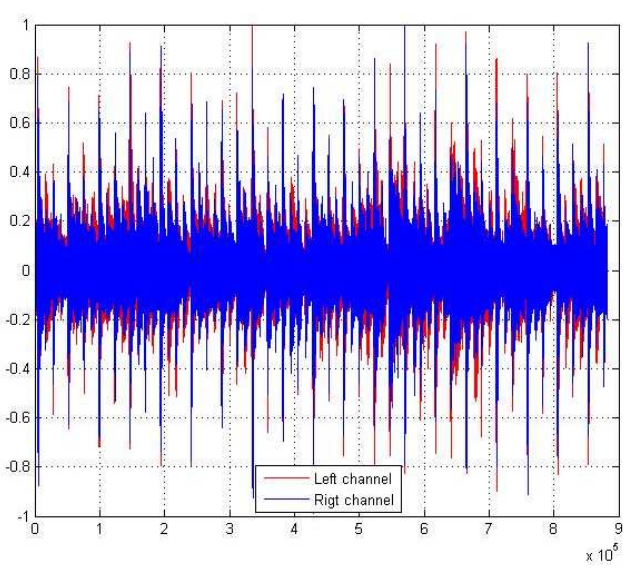

Fig. 1: Original Audio Signal

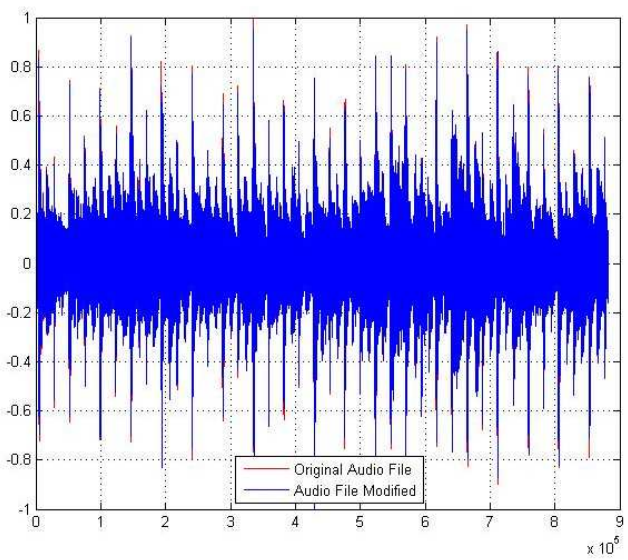

Fig. 2: Original Audio Signal and Audio Signal Modified

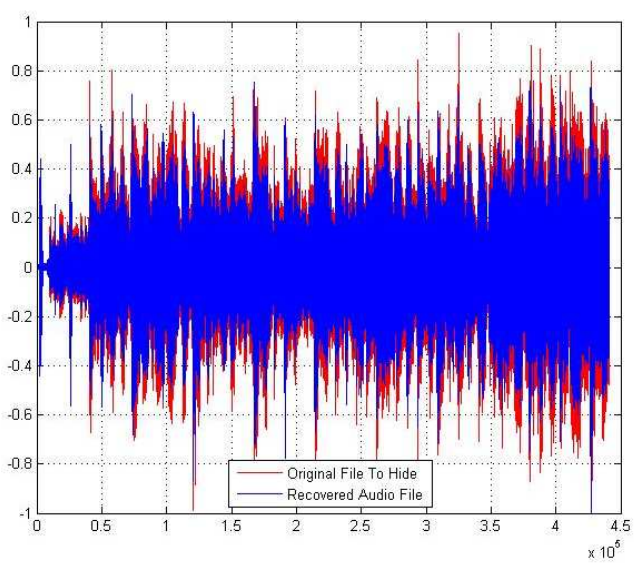

Fig. 3: Original Audio file and recovered audio file 
signals did not undergo major modifications, and they show correlation values above 0.9 . Furthermore, in most cases, the values of PSNR from the original signal with the signal extracted were above $30 \mathrm{~dB}$.

The columns distribution is the same as in Table 1; only the first column changed because we used fifty different host audio signals and the secret audio was always the same.

Graphically, the changes that took place on both host audio files, such as samples to hide, are shown in figures 4, 5 and 6 , respectively. We present the sample number 17. Figure 4 shows the sample which was utilized as host audio. While figure 5 shows the audio signal to hide. Finally, the original and extracted audio files can be seen in figure 6. It can be observed that magnitude variations of the signals did not alter the relevant information; changes are almost imperceptible. Both host and hidden files were clearly audible.

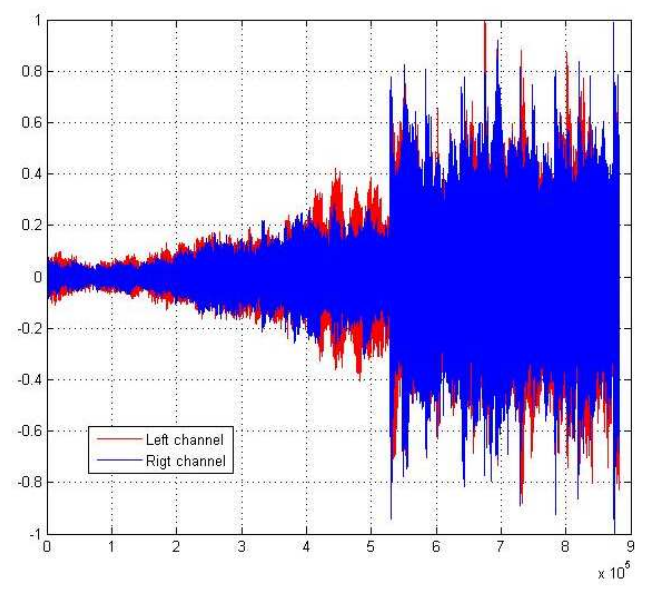

Fig. 4: Host Audio (sample 17)

Finally, in order to determine the efficiency of the used algorithm, Gaussian filter and Gaussian noise were applied on the host file. Since most of the results, regardless of the sample, are similar, we only display those of the sample 17, which was also used for the table 2 and the figures 4 , 5 , and 6 .

a) Gaussian Filter

Linear filtering was performed using a Gaussian filter, size $N x N$. The used average was $\mathrm{O}$, and the deviation standard 0.5. Both parameters remained constant during the test. Table 3 shows the correlation value that exists between the hidden file and the recovered one.

According to Table 3, it is clear that the hidden sample can be recovered in those cases in which the size of the Gaussian window is odd.
Table 2: Correlation and PSNR values (fifty different host samples)

\begin{tabular}{|c|c|c|c|}
\hline Host & $\begin{array}{c}\text { PSNR } \\
{[\mathrm{dB}]}\end{array}$ & $\begin{array}{c}\text { Correlation } \\
\text { carrier and } \\
\text { modified file }\end{array}$ & $\begin{array}{c}\text { Correlation } \\
\text { Original and } \\
\text { recovered file }\end{array}$ \\
\hline 1 & 33.1887 & 0.9908 & 0.9638 \\
\hline 2 & 31.0190 & 0.9958 & 0.9176 \\
\hline 3 & 30.7353 & 0.9860 & 0.9409 \\
\hline 4 & 30.6613 & 0.9930 & 0.9446 \\
\hline 5 & 37.2887 & 0.9972 & 0.9748 \\
\hline 6 & 40.8721 & 0.9987 & 0.9424 \\
\hline 7 & 37.1974 & 0.9981 & 0.8756 \\
\hline 8 & 35.7610 & 0.9972 & 0.9600 \\
\hline 9 & 31.7251 & 0.9968 & 0.8972 \\
\hline 10 & 31.1076 & 0.9849 & 0.9788 \\
\hline 11 & 36.0023 & 0.9968 & 0.9767 \\
\hline 12 & 42.7371 & 0.9986 & 0.9275 \\
\hline 13 & 30.6202 & 0.9950 & 0.9004 \\
\hline 14 & 36.4654 & 0.9961 & 0.9677 \\
\hline 15 & 30.8800 & 0.9933 & 0.9408 \\
\hline 16 & 32.6046 & 0.9973 & 0.8793 \\
\hline 17 & 40.2919 & 0.9986 & 0.9963 \\
\hline 18 & 35.8797 & 0.9952 & 0.9669 \\
\hline 19 & 36.0385 & 0.9714 & 0.9956 \\
\hline 20 & 38.2541 & 0.9985 & 0.9527 \\
\hline 21 & 34.5151 & 0.9948 & 0.9816 \\
\hline 22 & 28.4119 & 0.9829 & 0.9417 \\
\hline 23 & 31.4228 & 0.9916 & 0.9726 \\
\hline 24 & 30.5368 & 0.9964 & 0.9537 \\
\hline 25 & 41.2302 & 0.9991 & 0.9149 \\
\hline 26 & 33.7409 & 0.9984 & 0.8493 \\
\hline 27 & 33.1639 & 0.9950 & 0.9517 \\
\hline 28 & 32.8457 & 0.9241 & 0.9950 \\
\hline 29 & 33.0988 & 0.9934 & 0.9983 \\
\hline 30 & 49.6257 & 0.9995 & 0.9838 \\
\hline 31 & 34.6433 & 0.9984 & 0.9168 \\
\hline 32 & 33.4414 & 0.9980 & 0.8947 \\
\hline 33 & 33.8051 & 0.9984 & 0.9288 \\
\hline 34 & 36.1315 & 0.9952 & 0.8894 \\
\hline 35 & 26.8546 & 0.9740 & 0.9854 \\
\hline 36 & 36.0042 & 0.9916 & 0.9735 \\
\hline 37 & 30.9360 & 0.9974 & 0.8803 \\
\hline 38 & 32.7908 & 0.9879 & 0.9874 \\
\hline 39 & 36.2639 & 0.9923 & 0.9514 \\
\hline 40 & 29.4254 & 0.9947 & 0.8926 \\
\hline 41 & 37.0023 & 0.9974 & 0.9470 \\
\hline 42 & 32.5837 & 0.9956 & 0.9864 \\
\hline 43 & 38.9638 & 0.9971 & 0.9731 \\
\hline 44 & 39.6444 & 0.9989 & 0.8942 \\
\hline 45 & 33.0039 & 0.9952 & 0.9689 \\
\hline 46 & 34.5592 & 0.9961 & 0.9753 \\
\hline 47 & 30.7308 & 0.9936 & 0.9653 \\
\hline 48 & 34.0944 & 0.9957 & 0.9574 \\
\hline 50 & 32.0207 & 0.9928 & 0.9701 \\
\hline & 38.0587 & 0.9973 & 0.9570 \\
\hline
\end{tabular}




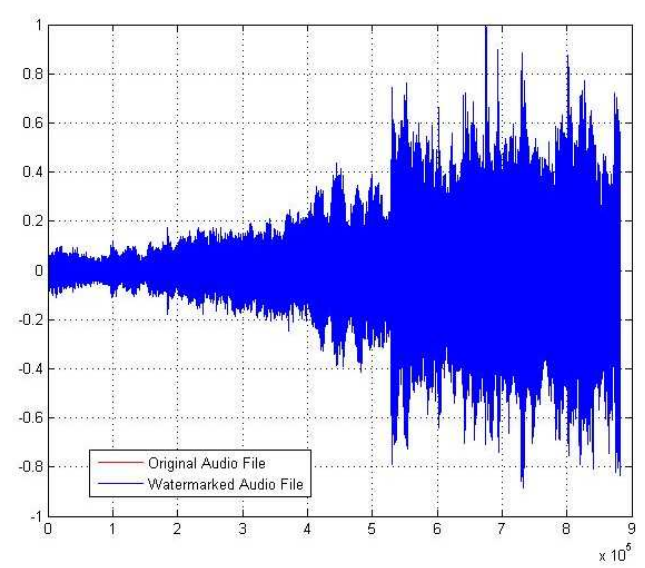

Fig. 5: Original Audio File vs Audio File Modified (sample 17)

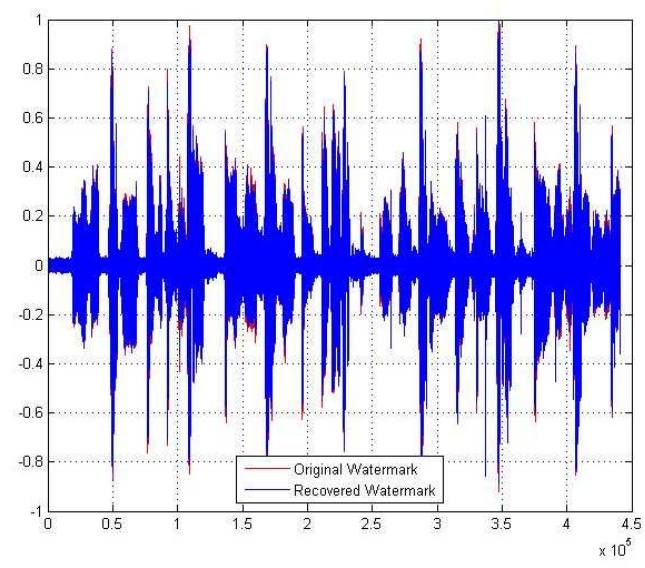

Fig. 6: Recovered Audio File

Table 3: Correlation values of the recovered file against the originally hidden file, after applying the Gaussian filter to the file with the concealed information.

\begin{tabular}{|c|c|c|}
\hline Sample & NxN & $\begin{array}{c}\text { Correlation } \\
\text { original and } \\
\text { extracted file }\end{array}$ \\
\hline & 2 & 0.0086 \\
\hline & 3 & 0.9902 \\
\hline & 4 & 0.0083 \\
\hline 17 & 5 & 0.9902 \\
\hline & 6 & 0.0083 \\
\hline & 7 & 0.9902 \\
\hline & 8 & 0.0083 \\
\hline & 9 & 0.9902 \\
\hline
\end{tabular}

Table 4: Correlation values of the recovered file against the original hidden file, after applying Gaussian noise to the file with the concealed data.

\begin{tabular}{|c|c|c|}
\hline Sample & $\begin{array}{c}\text { SNR } \\
{[\mathrm{dB}]}\end{array}$ & $\begin{array}{c}\text { Correlation } \\
\text { original and } \\
\text { extracted file }\end{array}$ \\
\hline & 0 & 0.0168 \\
\hline & 10 & 0.0491 \\
\hline & 20 & 0.1555 \\
\hline 17 & 30 & 0.4447 \\
\hline & 40 & 0.8415 \\
\hline & 50 & 0.9770 \\
\hline & 60 & 0.9943 \\
\hline
\end{tabular}

b) Gaussian Noise

Gaussian white noise was added, with diverse SNR values (Table 4). The correlation values obtained between the original embedded file and the one recovered for the different SNR values employed can be seen in Table 4.

Experimental results showed that, starting from a noise value of $40 \mathrm{~dB}$, it is possible to retrieve the sampled that was inserted in the host file. Moreover, the sample is audible clear.

\section{Conclusions}

This paper presents a steganography technique for audio files using the Hermite Transform (HT). An important feature of this method is that the information amount that can be concealment is half the total length of the audio file. Another contribution is the use of HT, that has proved to be useful in digital image applications (watermarking, image fusion, among other, etc.). One of the main advantages of the HT is the use of a Gaussian window for modeling the receptive fields of the human visual system (HSV).

The results of the experiments showed that the hidden audio file did not affect the quality of the original file. First, the hidden audio file was inaudible, and then, the values PSNR were above $30 \mathrm{~dB}$.

In those cases in which a Gaussian filter is applied onto the file with the hidden information, it can be recovered when the size of the filter window is odd. When Gaussian noise is added, even when the correlation values are low, the recovered sample can be audibly distinguished.

\section{Acknowledgements}

The authors are grateful to the anonymous referee for a careful checking of the details and for helpful comments that improved this paper. Sandra Gomez Coronel thanks to Universidad Nacional Autónoma de México grants IN113611 and IG100814 and Instituto Politécnico 
Nacional IPN (Comité Técnico para el Otorgamiento de Becas de Estudio, Apoyos Económicos y Licencias con Goce de Sueldo COTEBAL).

\section{References}

[1] Katzenbeisser, S., Petitcolas, F. Information Hiding, techniques for steganography and digital watermarking. Artech House, Computer security Series, (2000).

[2] Cox, I. J., Miller, M., Bloom, J., Fridrich, J., Kalker, T. Digital Watermarking and Steganography. San Francisco, CA. USA: Morgan Kaufmann Publishers Inc., (2007).

[3] Cvejic, N. Algorithms for Audio Watermarking and Steganography. MSc. Thesis, Department of Electrical and Information Engineering, Information Processing Laboratory, University of Oulu, Finland Oulu, (2004).

[4] Pickholtz, R. L., Schilling, D. L. and Millstein, L. B. Theory of spread spectrum communication a tutorial. IEEE transactions on Communications, 855-884 (1982).

[5] Martens, J. B. The Hermite Transform Theory. IEEE Transactions on Acoustics, Speech and Signal Processing, 38, 1595-1606 (1990).

[6] Escalante, B. López, P. and Silvan, J.L. SAR Image Classification with a Directional-Oriented Discrete Hermite Transform. Image and Signal Processing for Remote Sensing VII. Proceedings SPIE, 4885, 234-245 (2002).

[7] Bazziz, N. and Escalante-Ramiírez, B. and Romero, O. Image Watermarking in the Hermite Transform Domain with Resistance to Geometric Distortions. Proceedings of the SPIE. Optical and Digital Image Processing, 7000, 70000X, (2008).

[8] Young, R. The Gaussian Derivate Theory of Spatial Vision: Analysis of Cortical Cell Receptive Field Line-Weighting Profiles. Technical Report GMR-4920, General Motors Research, (1986).

[9] Kekre, H. B., Athawale, A., Swarnalata, B., Athawale, U. Increasing the capacity for the cover audio signal by using multiple LSBs for information Hiding. Third International Conference on Emerging Trends in Engineering and Technology, 196-201 (2010).

[10] Martens, J. B. The Hermite Transform Applications. IEEE Transactions on Acoustics, Speech and Signal Processing, 38, 1606-1618 (1990).

[11] Boas, R., Buck, R. Polynomial Expansions of Analytical Functions. New York: Springer, (1985).

[12] ConvertIt.com, Inc.: Handbook of Mathematical Functions with Formulas, Graphs and Mathematical Tables (AMS55), [on line], (2012). http://convertit.com/Go/ConvertIt/Reference/AMS55.ASP.

[13] Sanjit, K. M. Digital Signal Processing. McGraw Hill, New York, (2001).

[14] Álvarez. P. Ch. Identificar métricas para algoritmo que determina la mejor adaptación multimedia. Thesis. Escuela Politécnica Superior de Castelldefes, Catalunya, (2010).

[15] Siwar, R, H. and Sid-Ahmed, S. Audio Steganography Coding Using the Discreet Wavelet Transforms. International Journal of Computer Science and Security (IJCSS), 1, 79-93 (2011).

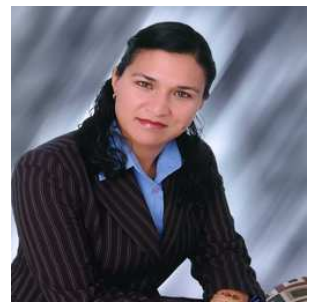

\begin{tabular}{ccr} 
Sandra & \multicolumn{2}{c}{ Gomez-Coronel } \\
Received her BS degree
\end{tabular} in Communications and Electronics Engineering from Escuela Superior de Ingeniería Mecánica y Eléctrica at Instituto Politécnico Nacional (IPN) in 2003, and her MSc degree in Telecommunications Engineering in 2008 at SEPI ESIME Zacatenco (IPN). She is currently a PhD student in Electrical Engineering at Universidad Nacional Autónoma de México. Her research interests include digital signal processing, specifically image processing. Also she has several years as bachelor professor at UPIITA (IPN).

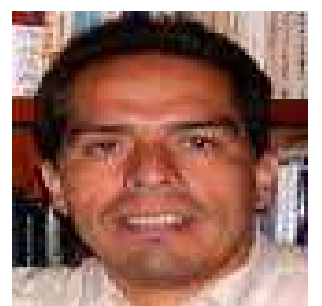

Boris Escalante-Ramírez $\mathrm{He}$ obtained the Engineer degree from the National University of Mexico in 1985, the M.E.E. degree from the Philips International Institute of Technological Studies, Eindhoven, The Netherlands, in 1987, and the Ph.D. degree from the Eindhoven University of Technology in 1992. He joined the Faculty of Engineering of the National University of Mexico in the same year. His research interests are computation models of human vision and their applications to image processing, including restoration, coding, fusion, watermarking, and optic flow.

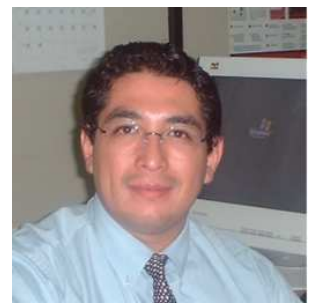

Marco Antonio Acevedo Mosqueda. He was born in Mexico City in July 19th, 1968. $\mathrm{He}$ received his BS degree in Communications and Electronics Engineering in 1992 and his MSc degree with specialization in Electronics in 1996 from Escuela Superior de Ingeniería Mecánica y Eléctrica at Instituto Politécnico Nacional. Currently, he is a professor in ESIME. His main research area is Digital Signal Processing and Telecommunications.

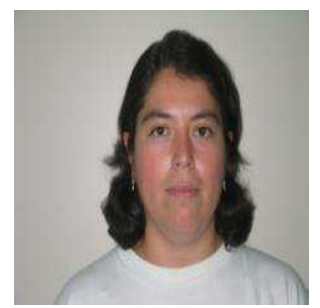

María Elena Acevedo Mosqueda. Received her BS degree in Engineering with specialization in Computing from the Escuela Superior de Ingeniería Mecánica y Eléctrica (ESIME) at Instituto Politécnico Nacional (IPN) in 1996. She has been teaching at ESIME since 1994. She received her MSc degree with specialization in Computing from the Centro de Investigación y de Estudios Avanzados (CINVESTAV) in 2001. She received her PhD from the Centro de Investigación en Computación (CIC) at IPN in 2006. Her main research area is Artificial Intelligence and Associative Memories. 